\title{
Solar Tracking Using Extended Mean Shift Based Color Histogram
}

\author{
Asepta Surya Wardhana ${ }^{1, *}$ Astrie Kusuma Dewi ${ }^{2}$ \\ 1,2Department of Instrumentation Refinery Engineering, Politeknik Energi dan Mineral Akamigas, Cepu-Blora 58315, \\ Indonesia \\ "Corresponding author. Email: aseptasw@gmail.com
}

\begin{abstract}
Nowadays, there are many solar tracking applications using photodiode sensors and Solar Position Algorithm. This tracking depends on the power of light and natural conditions. Inaccurate sun tracking causes the heat concentration to become weak and miss focus on heat-receiving objects. We developed a tracking algorithm to track the sun to support the control system of the dual parabolic concentrator. This algorithm is based on Extended Mean shift to find the tracking position of an object in a video sequence. This algorithm is effective since it exploits the estimation of kernel density for searching the local maximum of a similarity measurement of the color histogram. The Expectation Maximization algorithm functions to estimate model parameters and update the histogram display. An updated color histogram will improve the average shift tracking accuracy and reliability. We successfully applied this algorithm for solar tracking using 148 frames of data. In this experiment, the results obtained in the form of the average value of the color similarity of an object tracking with a truth tolerance percentage of $98.39 \%$.
\end{abstract}

Keywords: Tracking, Concentrator, Color Histogram, Extended Mean Shift.

\section{INTRODUCTION}

The big challenge for renewable energy progress is to provide effective solutions for environmentally friendly energy. One of them is the use of solar energy for the conversion of heat energy to electricity. Several applications such as the use of photovoltaic [1] and solar concentrator [2] have become a trend among the general public. However, all of these applications require solar tracking to support increased efficiency and optimize power output. In previous research on the dual parabolic concentrator, tracking was carried out using the cascade fuzzy algorithm and the Solar Position Algorithm which can produce very good accuracy so that the heat distribution on the receiver can be optimal 62.49\% [3]. The fuzzy method has been widely used in several applications that have been tested to be stable in controlling temperature systems and non-linear conditions [4], [5].

However, to support the existing tracking system, it is necessary to add other techniques to improve the performance of the system. The application of tracking using image processing has been applied to recognize images of the sun when covered in shadow [6] and combining image processing with LDR [7]. Lack of previous research, tracking is only in the form of images and not real time video. Processing of objects in the form of black white and gray image processing, this can make it difficult to recognize the sun's object if there is an obstacle. Non-rigid object tracking is an application to a variety of computer vision applications such as environmental monitoring [8], HCI [9] (humancomputer-interaction). Among all kinds of tracking algorithm, mean shift becomes popular because it is simple and robust [10]. The mean-shift algorithm is an idea invented by Fukunaga and Hostetler [11] for data cluster, which is referred as "valley-seeking procedure". Comaniciu and Meer [8] this research is successful for having image segmentation and tracking.

This paper gives new approach of a non-rigid object tracking by pursuant to mark the color and texture that give distribution characteristic of the object. The purpose of tracking is an approach for the wide variation of object with difference of color or texture so that it gives robust tracking facet which is difficult in measuring the rotation and the changing of scale.

The mean-shift algorithm operates by means of a probability distribution. To track a colored object in a sequence of video frames, the color image data must be 
represented as a probability distribution so that in this study we used a color histogram. A sequence of video images produces a color distribution that changes over time, so the mean-shift algorithm must be modified to adapt it dynamically to the probability distribution it tracks.

In this study, we use a color histogram-based tracking algorithm that estimates the position of the object being tracked, tracking is in the form of estimating an ellipse that approaches the shape of the object. The object can be estimated using the Expectation Maximization (EM) technique. An updated histogram capability for averaging shift tracker is able to track objects with a wide

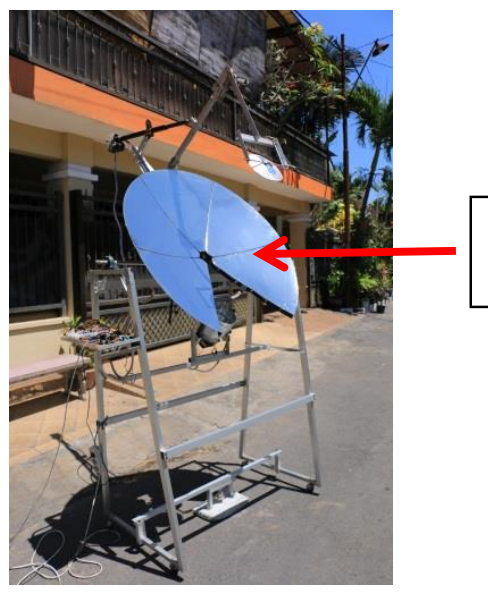

variety of views. We also place a value limit on the histogram to prevent tracking from drift. We applied our algorithms to solar tracking on Dual Parabolic Dish Concentrator. The experimental results show that our solar tracking algorithm can achieve a more robust and accurate tracking performance compared to the traditional mean-shift algorithm.

\section{METHOD}

The dual parabolic concentrator design used from the application research is presented in Figure 1. Sun tracking is used to move the main parabola so that it can position the incoming rays optimally.

Figure 1 Tracking application on dual parabolic dish concentrator. [3]

The solar tracking that is implemented uses several algorithms to be processed in video processing so that it can recognize the condition of the object to be observed. Some of the algorithms used are mean shift, Kalman Filter dan Expectation Maximization. The mean-shift tracking algorithm is a method based on the comparison of the original object histogram in the current image frame and the candidate area histogram in the next image frame. The results of this comparison are intended to maximize the correlation between the two histograms.

The color distribution is used as the target model because it is to achieve stability against non-rigid, partially closed and rotational conditions. The color distribution is shown in m-bins. The histogram is generated with the $h\left(x_{i}\right)$ function, which provides color information at the location $x_{i}$ to the appropriate bin. The histogram can be processed in RGB space. The color histogram is usually computed in RGB space using an $8 \times 8 \times 8$ bin. Suppose $\left\{x_{i}\right\}_{i=1 . . n}$ becomes the location of the target model pixel, centered at position 0 . The target model can be written in the equation [12]

$$
\hat{q}_{u}=C \sum_{i=1}^{n} k\left(\left\|x_{i}^{*}\right\|^{2}\right) \delta\left[b\left(x_{i}^{*}\right)-u\right]
$$

Where $\delta$ used as the Kronecker delta function. In this algorithm, the normalization constant $C$ is required to be derived by applying this condition $\sum_{i=1}^{m} \hat{q}_{u}=1$. Suppose $\left\{x_{i}\right\}_{i=1 . . n}$ is determined as the pixel location of the target candidate centered at position $y$. We use the same kernel $k$, radius $h$, the probability of color $u$ in the target candidate according to the equation [13].

$$
\hat{p}_{u}(y)=C_{h} \sum_{i=1}^{n_{h}} k\left(\left\|\frac{y-x_{i}}{h}\right\|^{2}\right) \delta\left[b\left(x_{i}\right)\right.
$$

For $u=1 \ldots m$

The $\hat{p}_{u}$ value is used as bin in kernel histograms where an index $u$ and $m$ are the number of bins. The value of $b\left(x_{i}\right)$ is the quantization of the pixel intensity at $x_{i}$. Kernel bandwidth which is the value of $h$ which normalizes the image coordinates so that the kernel radius becomes one. The normalization constant $C_{h}$ is used for the target candidate.

The similarity of the histogram determines an area of similarity to the object being observed. Bhattacharya coefficient is used in mean-shift to convert in convergence determination. The Bhattacharyy coefficient has a standard measure that is generally expressed between the two distributions $p(u)$ and $q(u)$. 


$$
\rho(y)=\rho[\hat{p}(y), q]=\sum_{u=1}^{m} \sqrt{\hat{p}_{u}(y) q_{u}}
$$

The value $\rho=1$ is identical to the condition of the two normalized histograms showing perfect similarity. So that the measurement can be expressed the distance between the two distributions [14]

$$
d(y)=\sqrt{1-\rho[\hat{p}(y), q]}
$$

From equation (4) is called the Bhattacharyya distance.

Where $C_{h}$ is constant factor, the weight function defined by [14]

$$
\omega_{i}=\sum_{i=1}^{m} \sqrt{\frac{\hat{q}_{u}}{\hat{p}_{u}\left(\hat{y}_{0}\right)}} \delta\left[b\left(x_{i}\right)-u\right]
$$

An identical Gaussian distribution is assumed to be used for all bins with variances. To get the value, use the mean-shift iteration as follows.

$$
\hat{y}_{1}=\frac{\sum_{i=1}^{n_{h}} x_{i} \omega_{i} G\left(\left\|\frac{\hat{y}_{0}-x_{i}}{h}\right\|^{2}\right)}{\sum_{i=1}^{n_{h}} \omega_{i} G\left(\left\|\frac{\hat{y}_{0}-x_{i}}{h}\right\|^{2}\right)}
$$

The value $\left\{x_{i}\right\}_{i=1 . . n}$ is a set of pixel locations around its center $\hat{y}_{0}$ in one size and $n_{h}$ frame which is affected by kernel bandwidth $h$. We use Kalman filter to achieve stability and robustness of object tracking by estimating the object's kernel histogram. So that it can maximize the estimation of the object model obtained. In this research, we carried out the model update equation according to the Kalman filter theory.

To calculate the Maximum Likelihood (ML) estimate in the presence of missing or hidden data, the EM (Expectation Maximization) Algorithm can be used as an efficient iterative procedure. Maximum Likelihood estimates the most likely model parameters for the observed data. The EM algorithm consists of two processes in carrying out iterations in the form of stages: E-step and M-step. In the (Expectation) E-step, the process of missing data is estimated based on the observation data and the latest estimation of the model parameters. In the (Maximization) M-step, we can maximize the lost data by performing the probability function. With the hope that the missing data estimate from the E-step process can be used as a substitute for the actual data lost.

In the previous process we can use the EM algorithm then it can be applied to the $\gamma$-normalized function. The conditions for the renewal equation remain the same. So the update equation for $V$ in step-M is given by:

$$
\begin{aligned}
& \vec{V}^{k+1} \\
& =\beta \sum_{i=1}^{N} q_{i}\left(\vec{x}_{i}\right. \\
& \left.-\vec{\theta}^{(k)}\right)\left(\vec{x}_{i}\right. \\
& \left.-\vec{\theta}^{(k)}\right)^{T} \\
& \text { Where } \beta=1 /(1-\gamma) .
\end{aligned}
$$

\section{RESULT AND DISCUSSION}

The test uses the extended mean shift algorithm to determine the robust aspect of changing the sun's movement from bottom to top. From Figure 2, ROI (Region of Interest) taking is carried out only on a small part of the object in the form of sunlight. The object is white and has diffuse light. The selection is made in accordance with the rules for taking the ROI by not exceeding the desired object because it can cause slow computation and chaotic tracking. ROI can be done in a variety of shapes (oval, cube, square, triangle and irregular).

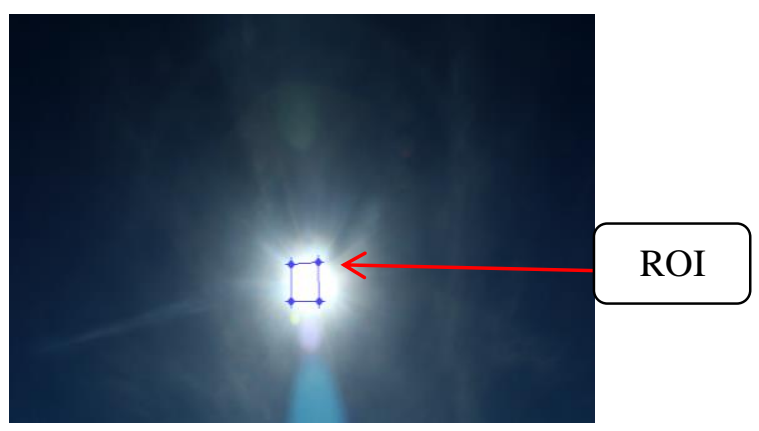

Figure 2 Experiment results in video of the sun at frame 2.

In this experiment, using the iterative algorithm 6 times with frame 2 to frame 150 . This system test is carried out on objects in the sun which are assumed to be moving from bottom to top or from morning to noon. From the experimental results in Figure 3, tracking at the beginning of frame 2 can be seen in the graph. The similarity value of rho $\rho$ measurement is between $1-0.95$ meaning that it still has a very good color distribution to maximize color. Tracking at the beginning of the frame has not shown a change in scale because the target is still modeling the initial distribution as a reference for further iterations. 


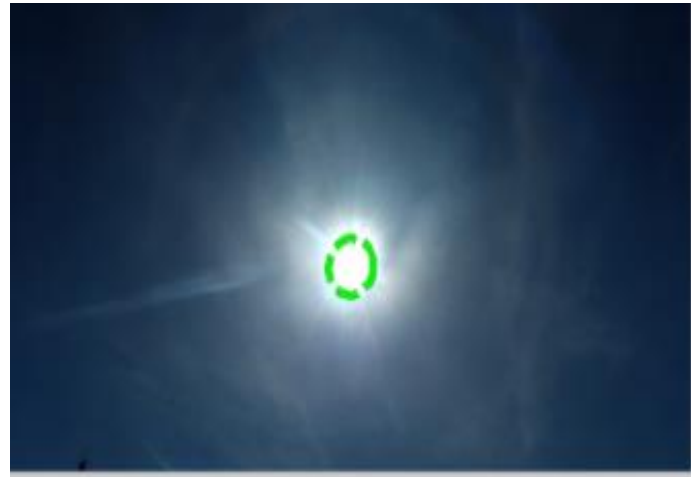

Experiment Result for Sun Solar tracking at Surabaya City

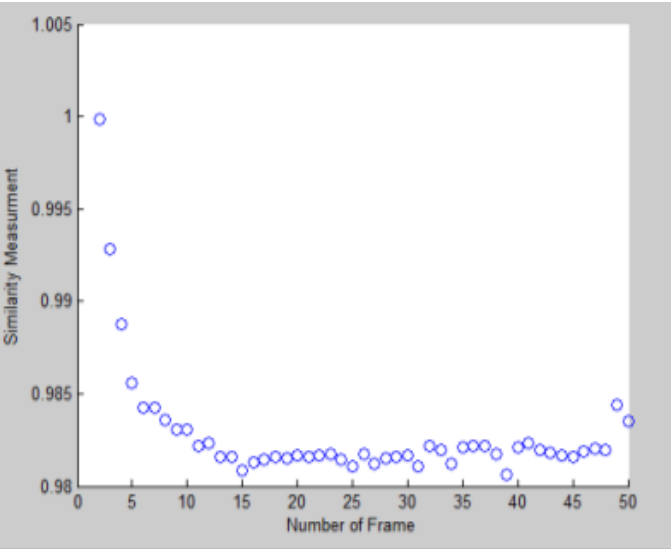

Figure 3 Experiment results in video of the sun at frame 2 until 50

The scale change will occur automatically following the size of the object that is recognized by the model and has the same color distribution. The scale variation decreases if there is no color distribution around the

Frame 2

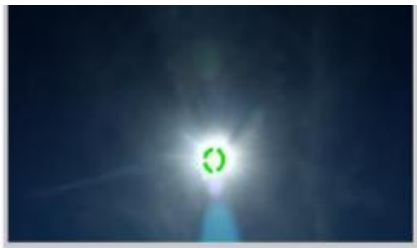

Frame 10

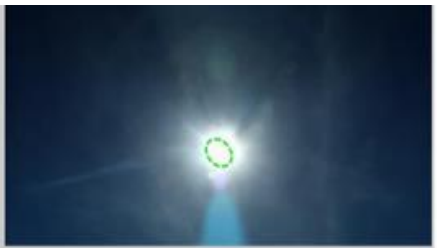

Frame 60

Frame 110

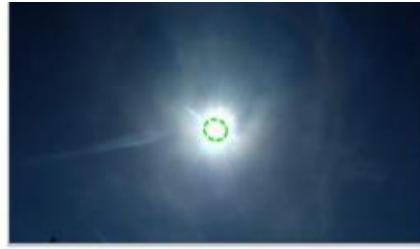

object that is the same as the model. Tracking until frame 50 , the object similarity value is obtained at $0.98-0.95$ or almost $98 \%$ of the objects can be recognized from the initial selection results of the ROI.

Frame 30

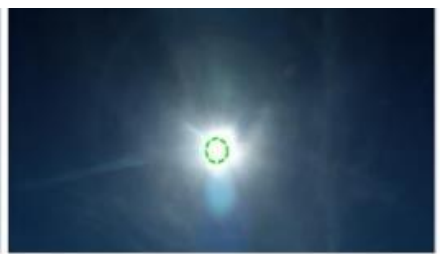

Frame 150

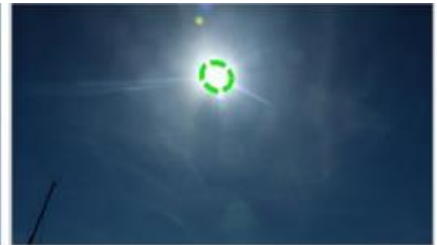

Figure 4 Solar tracking results from frame 2 until 150.

Tracking has succeeded in following the position of the sun from frame 2 until 150 with the position of the green circle still on the sun object as in Figure 4. This stability is achieved from the Kalman filter algorithm that can estimate measurements and correct the error for each covariance.

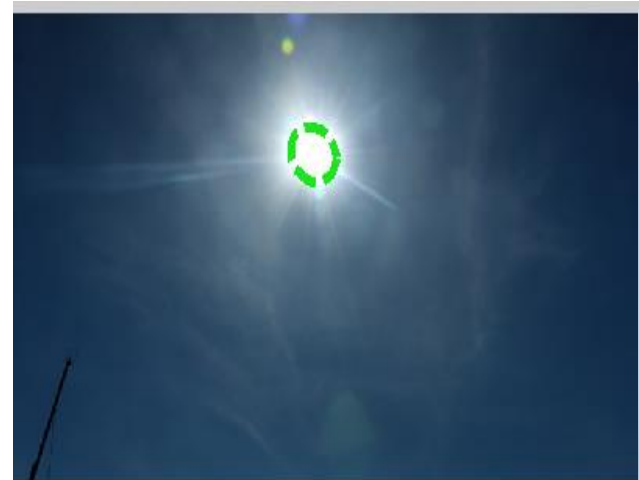

Experiment Result for Sun Solar tracking at Surabaya City

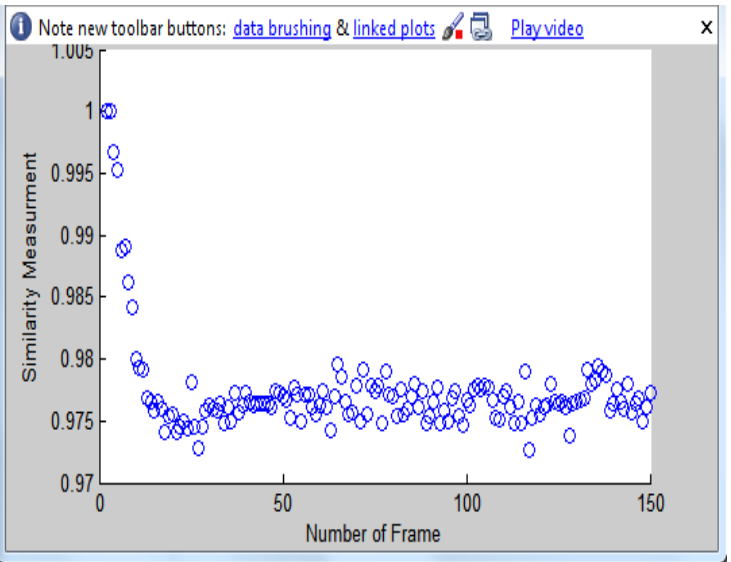

Figure 5 The result of the color similarity of all frames 
From the experimental results as in Figure 5 it can be taken the average value of the tracking results for frame 2 until frame 150 with the formula:

Average value $=$ Total number of measurements / number of frames

$=145,6237 / 148$

$=0,983943919$

The total number of measurements is the measurement value of the similarity of all frames. From the average value of the measurement similarity from tracking, the percentage calculation is as follows:

Truth tolerance percentage value $=0,983943919 \times 100 \%$ $=98,39 \%$

The calculation is done for data frame 2 until frame 150 with lots of data 148 pieces. From the experiments above, tracking can do more than $95 \%$ on average and is done well.

This research analyzes the Extended Mean Shift algorithm used in the experiment. In accordance with the planning in the previous chapter the equation of the algorithm is changed to. By adding the mean of the vector in the presence of the covariance matrices, the algorithm can estimate the measurement of two random variables into one variable.

If two variables tend to change together when one of them is above the expected value, then the other variables tend to also be above then the covariance between the two variables will be positive. Otherwise if one of them is above and below the expected value of covariance is negative. is the vector mean which is the model parameter of the color distribution. If is MLE (maximum likelihood estimator) for the value $\theta$.

The function of the scale variation using the covariance matrix is influenced by the value of $\beta$ in this experiment using 1 and 2 , which is the size of the tracking scale, if it is larger, the tracking area is not effective. Its ineffectiveness is unable to focus on objects and tracking becomes diffuse. On the contrary, if it is too small, the tracking will not be able to scale variations. For future research development, it will integrate video processing with the control system on the Dual Parabola Dish in real time.

\section{CONCLUSION}

This paper presents a tracking system for a sun object. The solar tracking algorithm using a color histogram can be integrated with the tracking system on the Dual Parabola Dish Concentrator to improve heat distribution in the receiver. The system employs the combination of variance scale of the extended mean shift with Expectation Maximization and Kalman filter for improving the tracking estimation. The system demonstrates robust tracking of a sun object in different situations. However, the programming complexity for the computation of one iteration in the algorithm is slightly higher than the computational complexity of the original mean-shift. For future research, we will improve the robustness and the performance of system.

\section{ACKNOWLEDGMENT}

The authors would like to express their gratitude to the Politeknik Energi dan Mineral Akamigas for their financial support for this research.

\section{REFERENCES}

[1] T. Wati, A. Sahrin, T. Suheta, and I. Masfufiah, "Design and Simulation of Electric Center Distribution Panel Based on Photovoltaic System," IOP Conf. Ser. Mater. Sci. Eng., vol. 462, no. 1, 2019.

[2] A. S. Wardhana, M. Ashari, and H. Suryoatmojo, "Designing and modeling a novel dual parabolic concentrator with three degree of freedom ( DOF ) robotic arm," Sol. Energy, vol. 194, pp. 436-449, 2019.

[3] A. S. Wardhana, M. Ashari, and H. Suryoatmojo, "Optimal Control of Robotic Arm System to Improve Flux Distribution on Dual Parabola Dish Concentrator," Int. J. Intell. Eng. Syst., vol. 13, no. 1, pp. 364-378, 2020.

[4] A. K. Dewi, A. A. B. A. Sahaya, and W. Sugiman, "Level and Temperature Monitoring System in Blending Process Using Zigbee Wireless Sensor Network," vol. 436, pp. 372-375, 2020.

[5] C. N. Hamdani, "Perancangan Sistem Kontrol Level Nonlinier Menggunakan Fuzzy-PID Supervision," INOVTEK - Seri Elektro, vol. 2, no. 1, p. 10, 2020.

[6] M. Abdollahpour, M. R. Golzarian, A. Rohani, and H. Abootorabi Zarchi, "Development of a machine vision dual-axis solar tracking system," Sol. Energy, vol. 169, no. July 2017, pp. 136-143, 2018.

[7] H. A. Sohag, M. Hasan, M. Khatun, and M. Ahmad, "An accurate and efficient solar tracking system using image processing and LDR sensor," 2nd Int. Conf. Electr. Inf. Commun. Technol. EICT 2015, pp. 522-527, 2016.

[8] M. Greiffenhagen, D. Comaniciu, H. Niemann, and V. Ramesh, "Design, analysis, and engineering of video monitoring systems: An approach and a case study," Proc. IEEE, vol. 89, no. 10, pp. 1498-1517, 2001 . 
[9] J. Krumm, S. Harris, B. Meyers, B. Brumitt, M. Hale, and S. Shafer, "Multi-camera multi-person tracking for EasyLiving," Proc. - 3rd IEEE Int. Work. Vis. Surveillance, VS 2000, no. November 2015, pp. 3-10, 2000.

[10] D. Liang, Q. Huang, S. Jiang, H. Yao, and W. Gao, "Mean-Shift Blob Tracking With Adaptive Feature Selection and Scale," Image (Rochester, N.Y.), pp. 369-372, 2007.

[11] K. Fukunaga and L. D. Hostetler, "The Estimation of the Gradient of a Density Function, with Applications in Pattern Recognition," IEEE Trans. Inf. Theory, vol. 21, no. 1, pp. 32-40, 1975.
[12] Y. S. Chia, W. Y. Kow, W. L. Khong, A. Kiring, and K. T. K. Teo, "Kernel-based object tracking via particle filter and mean shift algorithm," Proc. 2011 11th Int. Conf. Hybrid Intell. Syst. HIS 2011, no. 1, pp. 522-527, 2011.

[13] C. Yang, R. Duraiswami, A. Elgammal, and L. Davis, "Real-Time Kernel-Based Tracking in Joint Feature-Spatial Spaces," 2004.

[14] J. Fang, J. Yang, and H. Liu, "Efficient and robust fragments-based multiple kernels tracking," AEU Int. J. Electron. Commun., vol. 65, no. 11, pp. 915923, 2011. 